\title{
Design of Mental Health Education Management System for College
}

\section{Students}

\author{
Qin Huanchang ${ }^{1}$, Wang Meisuo* ${ }^{2, a}$ \\ ${ }^{1}$ School of Information Engineering, Baise University, Baise, Guangxi, 533000, China; \\ ${ }^{2}$ Health Center, Baise University, Baise, Guangxi 533000, China. \\ a13097765363@163.com \\ Corresponding author: Meisuo Wang, Health clinic, Baise University
}

Keywords: College Students; Mental Health Education; Management System

\begin{abstract}
With the development of society and technical level, the internet has become a new transmission mode, and gradually penetrates into people's daily production and life including mental health education in colleges. Mental health education management system is established to further improve the level of mental health education of college students. Besides, it helps college students solve their problems in daily learning and life, and effectively improve the mental quality of college students, thus laying a good mental basis for their future employment life. Based on the overview of mental health education management system, we expounded the design path of mental health education management system, thus further improving mental health level of college students.
\end{abstract}

\section{Introduction}

With the development of network technology level, the network education mode has gradually become a new educational management mode. The internet-based education management system can conduct targeted mental education for college students to break the limitation of previous mental health education to time and space, thus better adapting to the trend of modern social development. It is well known that the previous mental health education management model is mostly based on teacher management. Students passively accept the teacher's education. Based on this simple knowledge inheritance teaching management model, the students firmly grasp relevant knowledge points of mental health education to a certain extent. However, the initiatives of students in learning are ignored, which is not conducive to the achievement of teaching effectiveness ${ }^{[1]}$.

The establishment of college students' mental health education management system provides students with abundant mental health resources by using network education management platform based on traditional teaching mode. Besides, it overcomes the poor learning initiative of students in traditional education mode to a certain extent, strengthening the training of students' self-learning ability. The closer distance between teachers and students leads to effective communication between them. Therefore, the mental health education management system has become an effective place where the students are guided to explore mental knowledge.

\section{Summary of mental health education management system}

Mental health education management system is gradually developed based on the internet. With the 
development of scientific and technological level, the internet gradually penetrates into mental health education. In the network environment, mental health education system gradually develops into a new education mode. In this system, the network technology and teaching behavior levels are organically integrated to fully take advantages of network in mental health education. Various educational media are used to conduct mental health education management of students. In addition, it can achieve digital transmission of health education management. Through student-centered, non-face-to-face teaching management activities, the students can get more information from the network media by breaking linear management structure of traditional education.

Mental health education management system of college students mainly has characteristics including the following aspects. Firstly, network information technology is the media and method of mental health education management system. The realization of mental health education management system consists of the digitization of teaching resources and the establishment of new educational environment. Secondly, mental health education management system is also an intellectual activity of developing, processing and designing network education resources and information ${ }^{[2]}$. Thirdly, mental health education management system has the characteristics of privacy. In this virtual interactive network environment, students can express their own feelings by voluntarily exchanging their own values and the experiences in the process of mental health education. Teachers can conduct targeted mental health education according to the content expressed by the students. It is not necessary for the students to worry about the leakage of real names and genders in mental health education management system. By eliminating the mental burden, students can make equal and free mental health communication with teachers.

With high pertinence, mental health education management system achieves exchange of teaching information management technology with students through maintenance of computer networks, providing an effective way for individualization of mental health education ${ }^{[3]}$. High degree of freedom breaks the limitation of previous education model on time and space. Therefore, mental health education management activities in individual, group and mass are achieved to greatly improve the degree of freedom of mental health education for college students.

In this way, we can achieve the organic combination of mental health teaching activities inside and outside the school. The students can accept mental health education management with the network and mobile equipment anywhere and anytime. After logging in mental health education management system, the students can learn mental health education knowledge. In addition, the level of mental health education can be improved by repeating listening and watching the courses taught by some experts and teachers ${ }^{[4]}$.

\section{Necessity to establish mental health education management system for college students}

Firstly, the establishment of mental health education management system for college students meets the requirements of the times. With the development of society and economic information technology level, the social environment is becoming more and more complicated. These changes exacerbate the seriousness of college students' mental problems to a certain extent. Mental health education workers should understand how these mental problems occur and how to conduct mental health education of college students. Through mental health education management system, we can analyze mental health education problems of college students in depth. Combined with social culture, historical background and environmental factors, these problems can be solved by making perfect coping strategies ${ }^{[5]}$. In the past, the pedagogical and mental knowledge was used to conduct mental health education management of college students according to the actual situation of their mental development. This approach, to a certain extent, helps college students solve the problems in 
the process of growing and learning, promoting their mental quality and all-round development. However, this traditional teaching model has gradually deviated from the original intention. Mental health education management activities are gradually changed to the activities of teachers' teaching and students' knowledge integration. In a short period of time, students can master mental health theory. However, it is difficult to achieve mental health education effect without promotion of independent learning spirit ${ }^{[6]}$. Mental health education management system is established to strengthen students' autonomic learning abilities to a certain extent. Therefore, mental health education management activities are changed from the unilateral knowledge teaching to the interaction between teachers and students. Free, equal and open environment is created to improve the awareness of college students on mental health education, thus enhancing their emotional and moral qualities.

Secondly, the establishment of college students' mental health education management system conforms to the requirement of comprehensively promoting quality education. Since the late 1980s, the research of quality education has been conducted in China's education circles. Nowadays, quality education has become the guiding ideology of the whole education development. To a certain extent, the birth of quality education concept initiates us into the purpose and method of talent cultivation. We should adopt various educational modes, especially mental health education, to improve mental qualities of college students, thus achieving talent cultivation target. Only the students with good moral, physical and mental qualities can become the pillars of social development. The basic quality of a person plays an important role in the process of personal development. Good mental quality is the base of improving other qualities. Good mental quality can promote students' own development and the formation of other qualities. Recently, educators attach more importance to mental health education. Mental health educators should take responsibilities and make contributions for the realization of quality education. This target can be realized by the establishment of mental health education management system.

Thirdly, the establishment of college students' mental health education management system conforms to the people-oriented education idea. It is the prerequisite for the realization of mental health education management. It is also an effective way to improve the mental quality and health of college students. Mental health education manager should establish mental health education management system and people-oriented education model combined with campus network information technology and mental development characteristics of college students. In line with modern education concept, this teaching mode is the inevitable trend of health education development. Human is the abstract concept of mental health education. The object of mental health education management system is emotional, real people. In the process of education management, the essential problem is how people exist, live and explore the life. In this sense, the establishment of college students' mental health education management system aims at concerning college students. Therefore, it is necessary to establish people-oriented mental health education system for college students by deeply integrating into daily life and every stage of college students' growth and success ${ }^{[7]}$.

Finally, the establishment of college students' mental health education system is the requirements of strengthening and improving the ideological politics education of college students. It is well known that mental health education management for college students is an important part of moral education in colleges. It is also one of the effective ways to further improve the ideological and political education levels of college students. In 2001, the Ministry of Education promulgated The Opinion on Strengthening Mental Health Education for College Students. It definitely pointed out that strengthening mental health education of college students is an important part of moral 
education in colleges. Therefore, the status of mental health education in college moral education is confirmed. Mental health education and ideological politics education are two independent disciplines. However, they have common purpose-helping students solve mental problems by special mental knowledge and technology, thus maintaining the mental health of college students. There is certain difference between the working points of the two modes. However, the two modes gradually close up to form the situation of mutual promotion and dependence with the advent of internet era and the establishment of mental health education management system. Practices show that there is close relationship between ideological politics quality cultivation and mental health education. Good ideological politics quality leads to the improvement of mental health level. A person with good mental health level can also accept the ideological politics education in a better mental state.

\section{Influencing establishment factors of mental health education management system for college students}

We should take full account of various influencing factors, such as the components and college students' mental acceptance process in mental health education management system.

Components of Mental Health Education Management System of College Students. The operation procedure of mental health education management system of college students is mainly composed of subject, object and content. For mental health education management system, the subject consists of the education workers and college students; the medium is the campus network platform; the object the mental health education content. These three aspects are organically integrated to gradually form mental health education management system through interaction in specific operation process.

Mental Acceptance Process of College Students in Mental Health Education Management System. The object and content of mental health education management are transferred to the subject through the medium. In the process of accepting mental health education management, positive and negative information of the subject will be fed back through a certain channel. Mental health education managers are predominant in mental health education management system. By determining and processing the feedback information, the object is adjusted to better assist education target.

Establishment Strategy of Mental Health Education Management System for College Students. While establishing mental health education system for college students, mental health education workers should pay attention to the following aspects.

(1)Attention to information

According to psychology, the attention is divided into structural and functional attention caused by external stimuli and subjective state, respectively. External stimuli and demand fit are the main factors that lead to the above two kinds of attention. Therefore, these two elements should be emphasized to establish mental health education management system for college students. Based on the actual situation of college students' mental health education, we should scientifically adjust the proportions of the two elements to further optimize the effect of mental health education management.

(2) Keeping information

People often pay little attention to common information, except for the information they are interested in. Therefore, it is necessary to attach great importance to the maintenance of information. The receiver's interest and external stimulus are organically integrated to enable college students to 
permanently concern mental health education management work, laying foundation for students' acceptance in the future.

(3) Receiving information

The process of receiving information is a systematic process, which includes information interpretation, screening and integration. In the process, each link is very important. Once one of the links is wrong, then the information will be hardly accepted by students and internalized into their own values or value orientations. Information interpretation consists of intentional interpretation of transitional information and the interpretation of relevant self-information. While establishing mental health education management system, we should enable college students to understand the intention and positive effect of mental health education on self-development, thus improving students' acceptance.

(4) Heart internalization

Relevant research results show that any information is acquired by internalizing the external information into self requirement. According to different information characteristics, people break the original recognition to establish a new cognitive structure. Therefore, college students gradually internalize educational theory into their own value orientations after receiving mental health education. This is the main purpose of heart internalization. Through this link, college students can change external theory knowledge to value orientation conforming to social need and self-development.

(5) Changing cognition

The educational theory knowledge is internalized into self-value tendency to probably change the original cognitive structure, thus gradually forming a new structure. After that, the values can be formed by treating surrounding people and things according to new cognitive structure.

The above points with close relationship should be considered in the establishment of mental health education management system for college students. Once ignoring one of these factors, we will fail to establish mental health education management system and to achieve the desired effect. 


\section{Establishment of mental health education management system for college students}

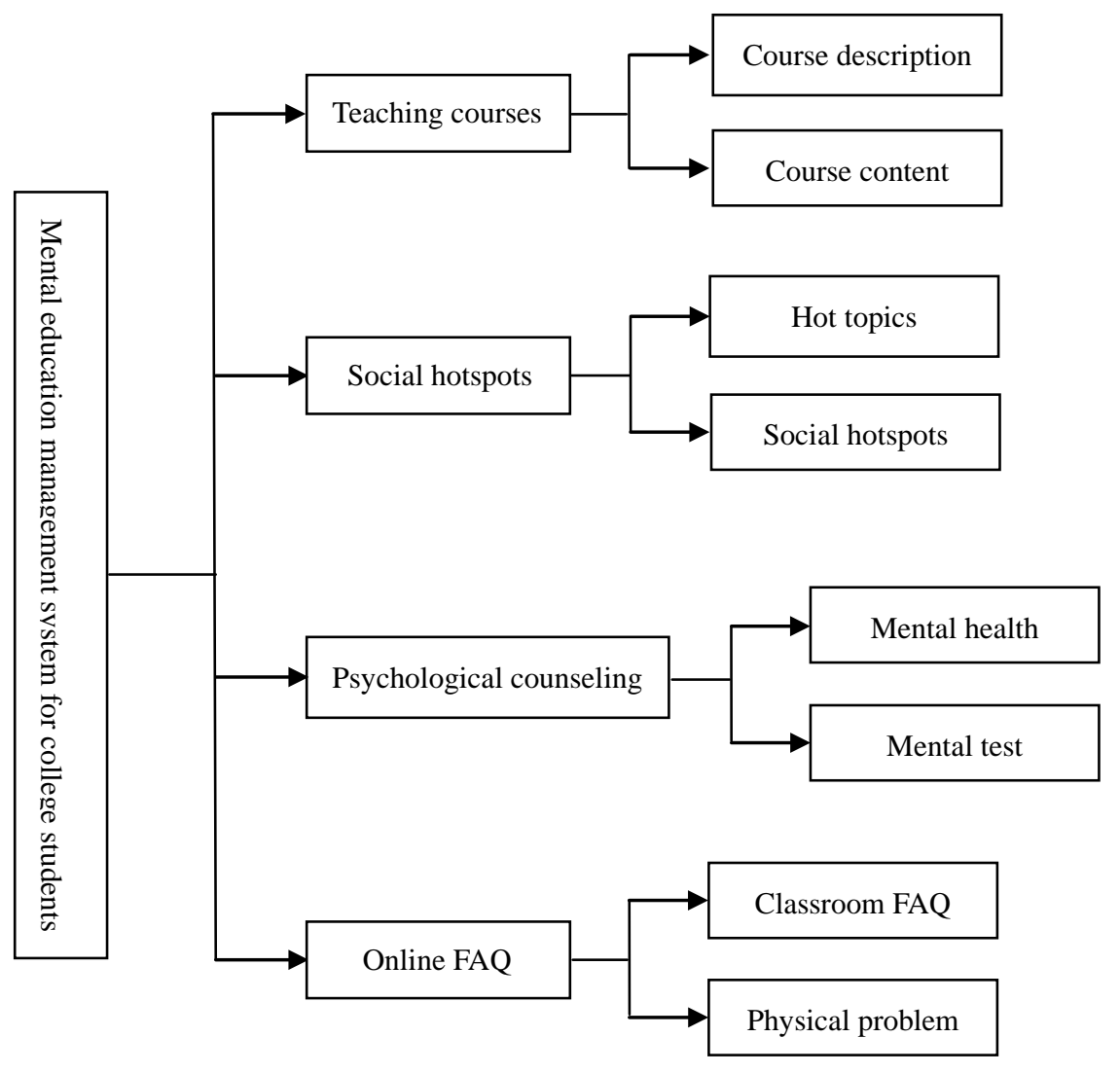

Figure 1 Structure of mental health education management system for college students

The advantages of network multimedia are fully used in establishment content design of mental health education management system for college students. The students are provided with good mental health education network environment through organic combination of multimedia images and sounds. Figure 1 shows the structure diagram of mental health education management system for college students. Figures 2 are the main interfaces of management system.
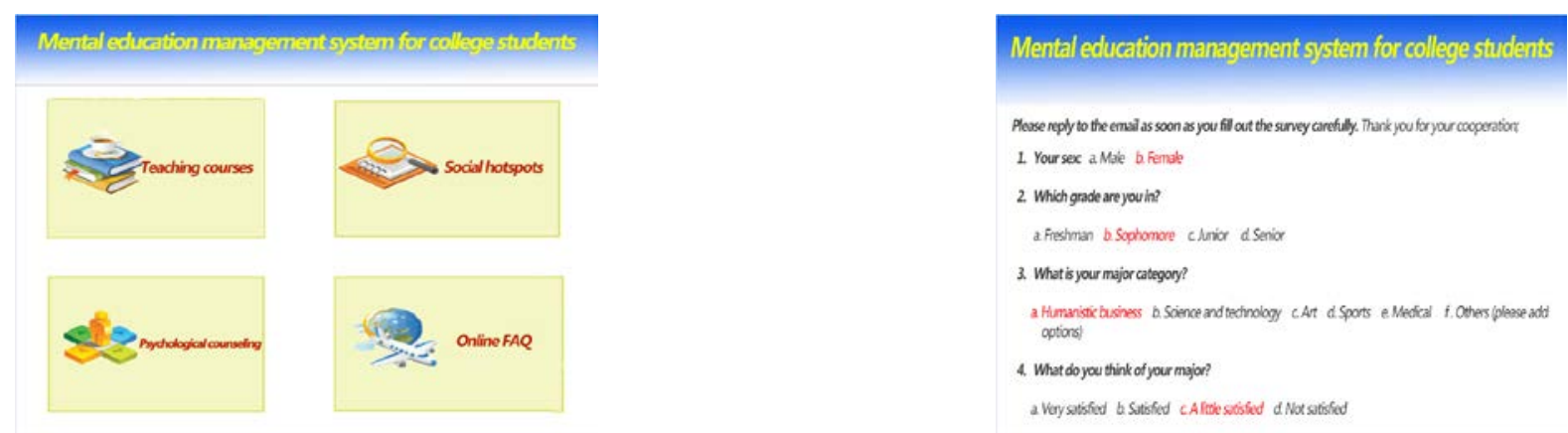

Figure 2 Logon interface of mental health education management system for college students

\section{Conclusions}

Consequently, the establishment of mental health education management system for college students is of great significance to the mental health promotion of college students and 
implementation of quality education concept. Therefore, various measures should be taken to establish mental health education management system for college students, thus laying foundation for the promotion of students' mental health. The mental health education management system is not built overnight. As a systematic process, it requires joint efforts of relevant educators.

\section{Acknowledgement}

The work was supported by Baise University Engineering Master Construction Project Start Fund (Computer Technology Application); the research achievement of Guangxi University Science \& Technology Research Project, Mental Health Problems and Educational Research of Minority College Students in Social Transformation Period (Project No.: YB2014398).

\section{References}

[1] Luo Liangliang, Mental File Management System for College Students, Nanchang University, 2008.

[2] Zhao Wenli, Design and Development of Auxiliary Education System of Network Mental Health for College Students, Shanghai Normal University, 2009.

[3] Wang Xiaoya, Design and Development of Auxiliary Education System of Mental Health for Artistic Undergraduates Based on Web, East China Normal University, 2010.

[4] Sun Xiaofeng, Innovation of Mental Health Education for College Students in China, Hebei Normal University, 2008.

[5] Design and Implementation of Mental Health Assessment System for College Students Based on Fuzzy Judgment, Yanshan University, 2012.

[6] Yang Yurong, Design and Implementation of Auxiliary Teaching Platform of Mental Health for Students in Higher Vocational Colleges, Hunan University, 2013.

[7] Yu Yingrong, Design and Implementation of Mental Health Education Management System in Weifang Medical University, Shandong University, 2011. 